\title{
Un periodo sismico nella zona dei Monti della Tolfa
}

\author{
A seismic period in the area of the Tolfa-Mountains
}

M. DE PANFIris

Ricevuto il 18 Ottobre 1969

Riassunto. - Il 2 Luglio 1969 , alle $09^{\mathrm{n}} 56^{\mathrm{m}}$ circa dell'ora legale $\left(07^{\mathrm{n}} 56^{\mathrm{m}}\right.$ dell'ora di Greenwich), una intensa scossa di terremoto, seguita da alcune repliche minori, ebbe luogo nei Monti della Tolfa, fra il Lago di Bracciano e il litorale civitavecchiese. Il fenomeno fu particolarmente interessante per essersi manifestato in una zona apparsa fino ad oggi sismicamente ab. bastanza tranquilla.

Nella presente nota, sulla base di accurate indagini effettuate nell'area più fortemente colpita ed in tutta la regione dove il movimento tellurico si rese percettibile alle persone, sono state determinate le coordinate dell'epicentro ed è stata rappresentata la propagazione macrosismica della scossa principale mediante la rete completa delle isosiste, da quella di grado massimo (VII) a quella di grado minimo (II).

Della scossa principale, con i dati ricavati dai sismogrammi ottenuti negli Osservatorî di Roma e di Monte Porzio Catone, sono state calcolate la profondità ipocentrale e l'ora origine.

Segue una descrizione dell'attività sismica nella regione che si estende intorno alla zona dei Monti Tolfetani.

SUmary. - On July 2nd, 1969, at about $07^{\mathrm{n}} 56^{\mathrm{m}}$ (Greenwich Mean Time), an intense earthquake shock, followed by a series of minor ones, occurred in the Tolfa Mountains, located between the Bracciano Lake and the littoral of Civitavechia. The phenomenon was of a particular interest, since it occurred in an area that until present days had seemed to be rather quiet seismically.

With the present note, on the base of thorough investigations conducted in the hardest-hit area and in all places where the seismic movement had been perceivad by the inhabitants, the coordinates of the epicenter are determinated and the macroseismic propagation of the main shock is represented with the map of isoseismical lines. 
With the data obtained from seismograms recorded by the stations of Rome and of Monte Porzio Catone, the hypocentral depth and the origin time of the main shock have been calculated.

This is followed by a description of the seismic activity in the region extending around the area of the Tolfa Mountains.

\section{1. - INTROdUzione.}

La mattina del 2 Luglio 1969 l'Istituto Nazionale di Geofisica di Roma fu più volte chiamato al telefono dalle Autorità di numerosi paesi situati a Nord-Ovest della Città; si chiedevano notizie circa alcuni movimenti tellurici che, ove più ove meno intensamente, in qualche luogo con vivo spavento della popolazione, erano stati avvertiti in molte località del Braccianese, del litorale tirrenico intorno a Civitavecchia e della zona dei Monti della Tolfa. L'Istituto di Geofisica poteva rispondere comunicando quanto, pochi minuti avanti, era stato rilevato in un primo esame delle registrazioni sismografiche ottenute negli Osservatorî di Roma e di Monte Porzio Catone dell'I.N.G.: una forte scossa di terremoto, di magnitudo 4.31, si era verificata alle $9^{\mathrm{n}} 56^{\mathrm{m}}$ circa (ora legale) ad una cinquantina di chilometri da Roma, in direzione Nord-Ovest; l'epicentro, per quanto ciò potesse apparire singolare considerando la modestissima sismicità della zona, risultava, a questo primo esame, ubicato nel gruppo montuoso della Tolfa o nelle sue immediate vicinanze. Alla scossa delle $9^{\mathrm{h}} 56^{\mathrm{m}}$ avevano fatto seguito, a brevi intervalli di tempo, numerose repliche di minore intensità.

I giornali pomeridiani del 2 Luglio e quelli antimeridiani del giorno successivo riportarono, con vistosi titoli, notizie piuttosto allarmanti circa le conseguenze del movimento tellurico: sgombero di edifici pericolanti a Civitavecchia, crolli parziali e gravi danni in alcuni paesi dell'Alto Lazio, scene di panico generale, tendopoli sorte sulle spiagge tirreniche e nelle campagne. Tali notizie peraltro apparivano poco concordanti con quelle pervenute direttamente all'Istituto e, in ogni caso, insufficienti a dare una chiara idea sia sulla gravità delle scosse sia, soprattutto, sulla propagazione del movimento tellurico attorno alla zona epicentrale indicata.

Pertanto, nei giorni 4 e 5 Luglio, lo scrivente si recava nelle zone maggiormente colpite dai sismi e poteva così constatare che lo spavento delle popolazioni, in molti centri abitati, aveva effettivamente raggiunto punte molto elevate, ma che i danni, eccetto 
in una piccola zona intorno all'epicentro, erano stati in verità assai modesti. Le notizie sparsesi nelle prime ore successive alle manifestazioni sismiche, controllate sul luogo, apparvero alquanto esagerate. In Civitavecchia, è vero, erano stati fatti sgomberare d'autorità alcuni fabbricati, ma trattavasi di edifici già lesionati da molto tempo e che le scosse, pur non molto intense nella città, avevano posto in preoccupanti condizioni d'instabilità.

La zona laziale compresa fra la costa tirrenica e il Lago di Bracciano, ove è stata individuata l'area epicentrale del terremoto del 2 Luglio 1969, si presenta oggi forse per la prima volta nella storia sismica italiana. E da ritenere per certo che tale territorio, in tempi antichissimi, quando esso veniva lentamente emergendo dal mare ed era intensa l'attività vulcanica che doveva portare alla formazione delle colline trachitiche tolfetane, sia stato scosso da violenti movimenti tellurici; non abbiamo però notizie di terremoti corocentrici di una qualche rilevanza che, in tempi storici, ne abbiamo turbato la quiete. Nella Carta sismica edita dal Baratta nel 1936, esso è rappresentato perció come una delle zone d'Italia che possono praticamente essere considerate come asismiche.

Il presente studio sul terremoto del 2 Luglio 1969 può pertanto essere utile quale contributo ad una completa conoscenza delle aree sismiche della regione laziale.

\section{2. - EPicentro E zona EPICENTRALE.}

Il breve periodo sismico che è argomento di questa nota si svolse quasi completamente durante la giornata del 2 Luglio 1969. La scossa iniziale, avvenuta alle $09^{\mathrm{h}} 56^{\mathrm{m}}$ circa $\left(^{*}\right)$, fu quella di maggiore intensità e raggiunse il VII grado della Scala Mercalli. Ad essa, dopo pochi minuti, segui una replica di V-VI grado; poi, durante la stessa giornata, altre nove scosse tutte d'intensità inferiore al $\mathrm{V}$ grado. Una replica leggera ebbe luogo la mattina del 3 Luglio; un'altra, leggerissima, avvertita solo nella zona epicentrale e non registrata dagli Osservatori di Roma e di Monte Porzio, nelle prime ore del giorno 5; l'ultima, pure leggerissima, alle $22^{\mathrm{h}} 50^{\mathrm{m}}$ circa del 9 Luglio.

(*) I tempi qui indicati si riferiscono all'ora legale adottata in Italia dal $1^{\circ}$ Giugno al 27 Settembre 1969. I tempi corrispondenti all'ora di Greenwich si avranno togliendo due ore dai tempi indicati. 
Ecco l'elenco delle scosse dell'intero periodo con l'indicazione, per ciascuna di esse, dell'ora di registrazione nell'Osservatorio di Roma, dell'intensità in gradi Mercalli e della magnitudo calcolata nell'I.N.G..

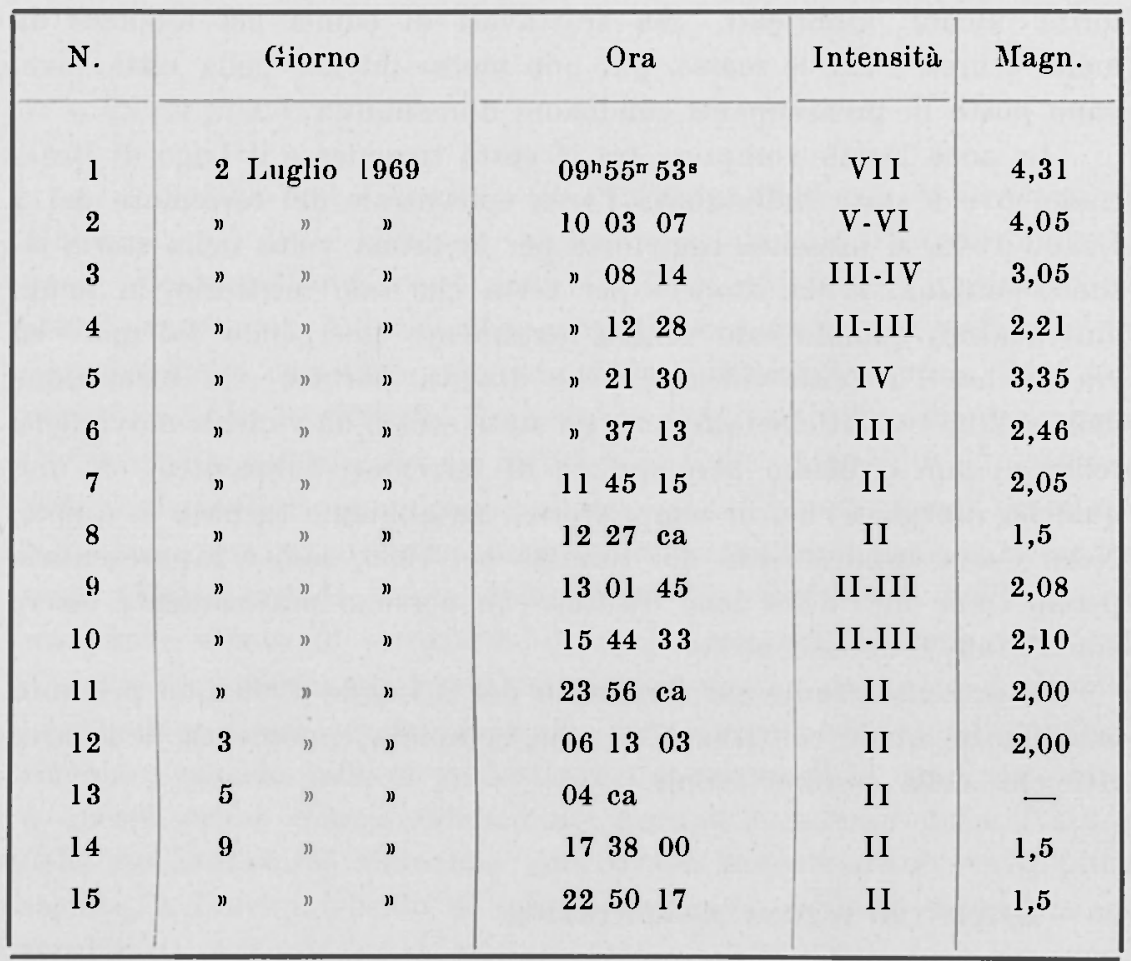

Subito dopo le scosse del 2 Luglio, come si è dianzi accennato, vennero fatti dei sopralluoghi dapprima nelle località da cui erano giunte notizie di danni e poi anche in quelle dove i movimenti tellurici erano stati sentiti con minore intensità. Accurate indagini macrosismiche, i cui risultati sono riassunti dalle linee isosismiche rappresentate nella Fig. 1, hanno permesso di precisare la posizione dell'area epicentrale e di stabilire i limiti della regione entro la quale la scossa principale si rese sensibile alle persone.

La zona epicentrale, colpita dalla scossa delle $9^{\mathrm{h}} 56^{\mathrm{m}}$ con un'intensità di VII grado, si estende ai due lati della media valle del Fiume Mignone, notevole corso d'acqua che, originatosi dal M. Termini, nella parte nord-occidentale dei Monti Sabatini, col suo andamento tortuoso cinge a levante e settentrione i Monti della Tolfa e, dopo aver ricevuto il 


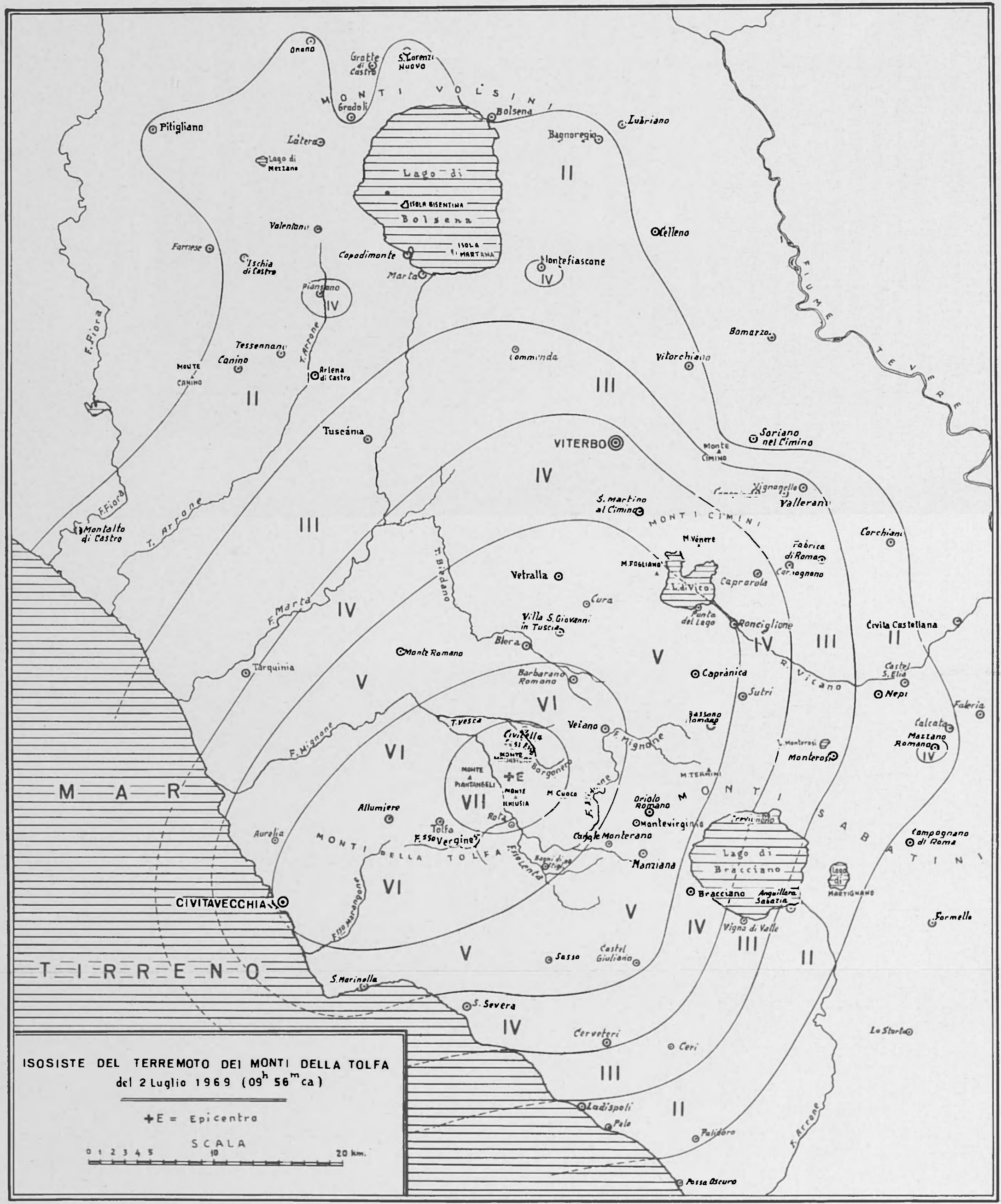

Fig. 1 
tributo di numerosi affluenti a carattere torrentizio (localmente chiamati "fossi "), giunge al Tirreno in prossimità delle saline di Tarquinia.

In questa zona, in cui affiorano estese formazioni flyshoidi di età cretaceo-oligocenica e potenti assise di argille gessifere del Mio-Pliocene sulle quali si sovrappongono qua e là, sotto forma di lembi isolati ignimbritici o di cupole laviche, le vulcaniti del massiccio eruttivo tolfetano, si trovano, oltre a case coloniche sparse, due centri abitati distanti l'uno dall'altro circa otto chilometri in linea d'aria: Rota, frazione del Comune di Tolfa, e Civitella Cesi, frazione del Comune di Blera.

Rota è un piccolo borgo, oggi abitato da poche decine di abitanti, che sorge a quota 191 sul mare sopra un grande masso di roccia vulcanica pianeggiante in sommità e situato alla confluenza del Fosso Verginese col Fiume Mignone. Il nucleo edilizio comprende un castello le cui origini risalgono al XIV secolo, un modesto gruppo di case d'abitazione ed una chiesa secentesca dedicata a S. Girolamo, protettore del Borgo.

Questa località è stata fortemente danneggiata dal terremoto del 2 Luglio 1969. Alla scossa delle $9^{\mathrm{n}} 56^{\mathrm{m}}$ è crollata la parte superiore

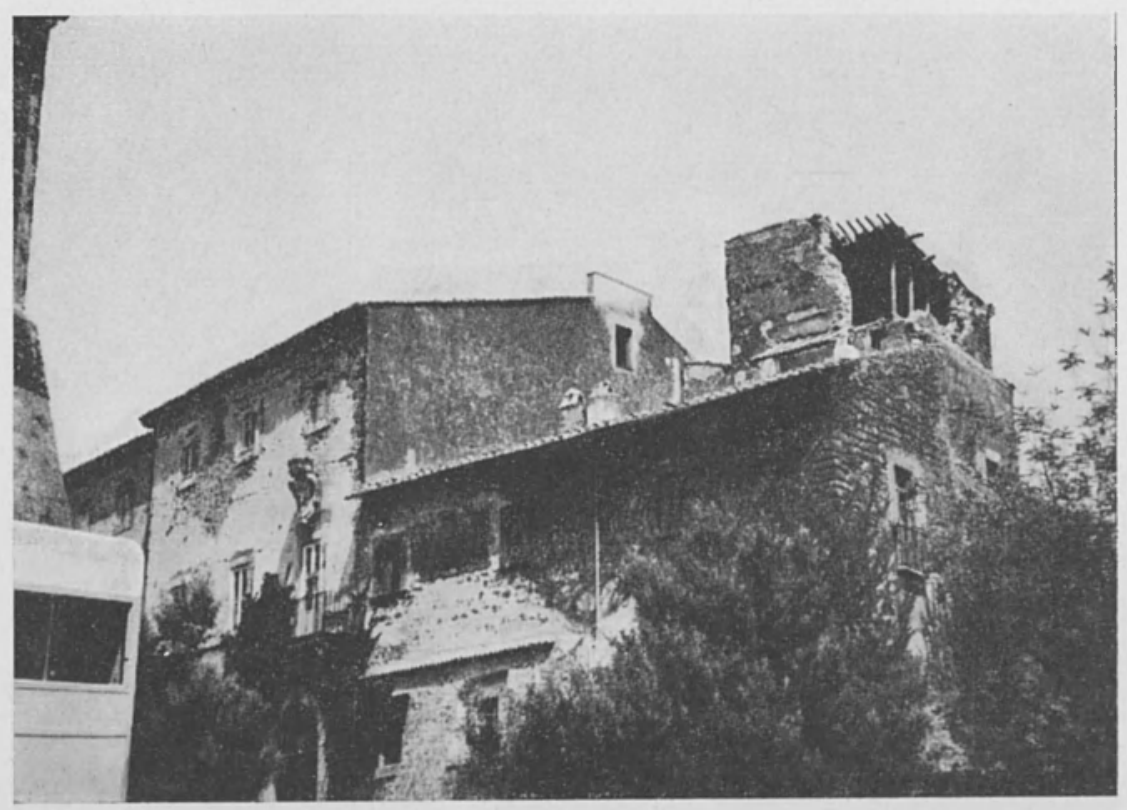

Fig. 2 - Facciata del castello di Rota. In alto, a destra, la torre in parte crollata per effetto del sisma.

(Foto G. Cirilli) 


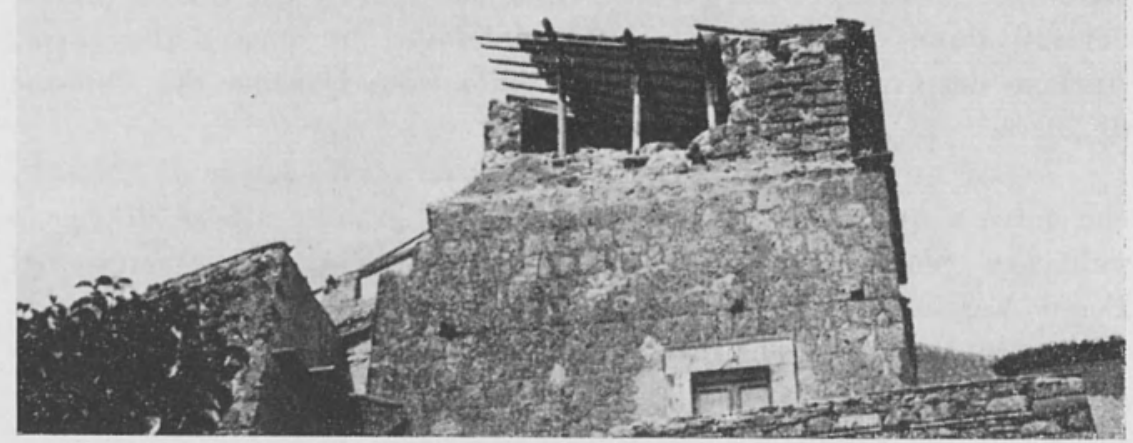

Fig. 3 - Veduta laterale della torre del castello di Rota. La parte inferiore, di costruzione trecentesca, è rimasta indenne; quella superiore, di costruzione posteriore, è crollata quasi totalmente.

(Foto G. Cirilli)

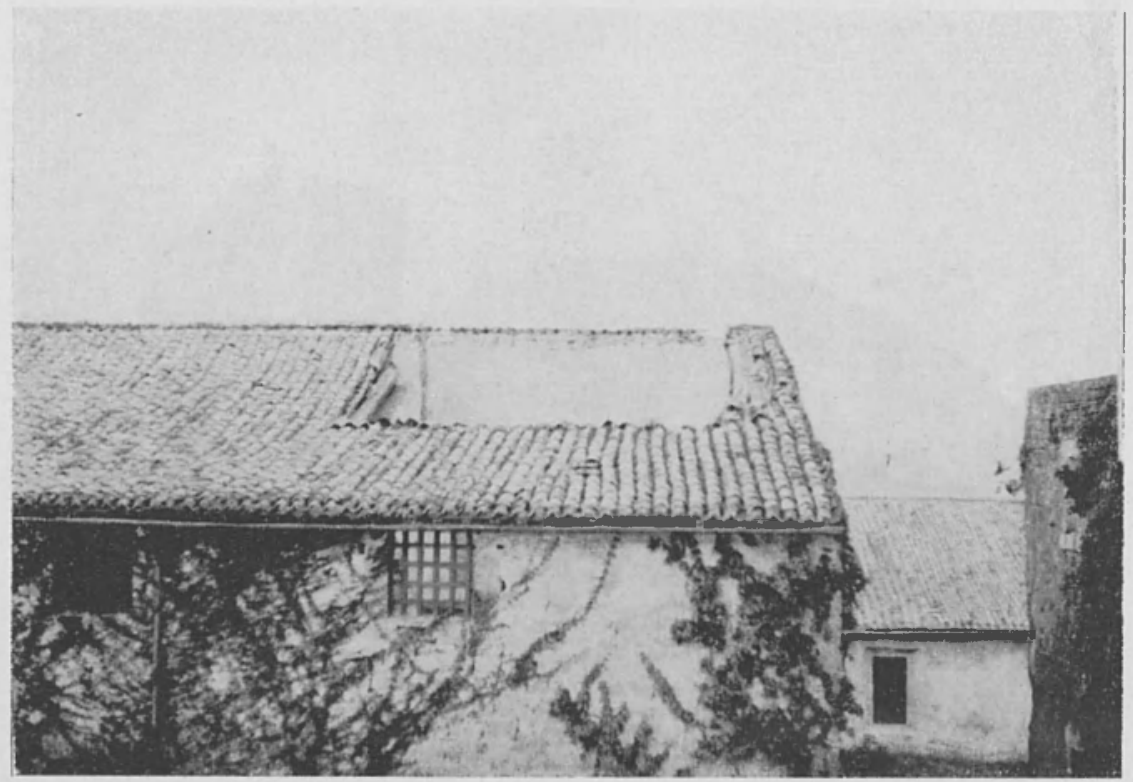

Fig. 4 - Crollo parziale del tetto di un edificio annesso al castello di Rota. (Foto G. Maiolo) 
della torre trecentesca del castello (Figg. 2 e 3), è sprofondato il tetto di una vicina costruzione adibita a granaio (Fig. 4) e un blocco di roccia si è distaccato dalla rupe su cui poggia l'abitato precipitando nel greto del Verginese. Nell'interno del castello le volte di alcune sale sono rimaste seriamente lesionate (Fig. 5), numerose ed ampie

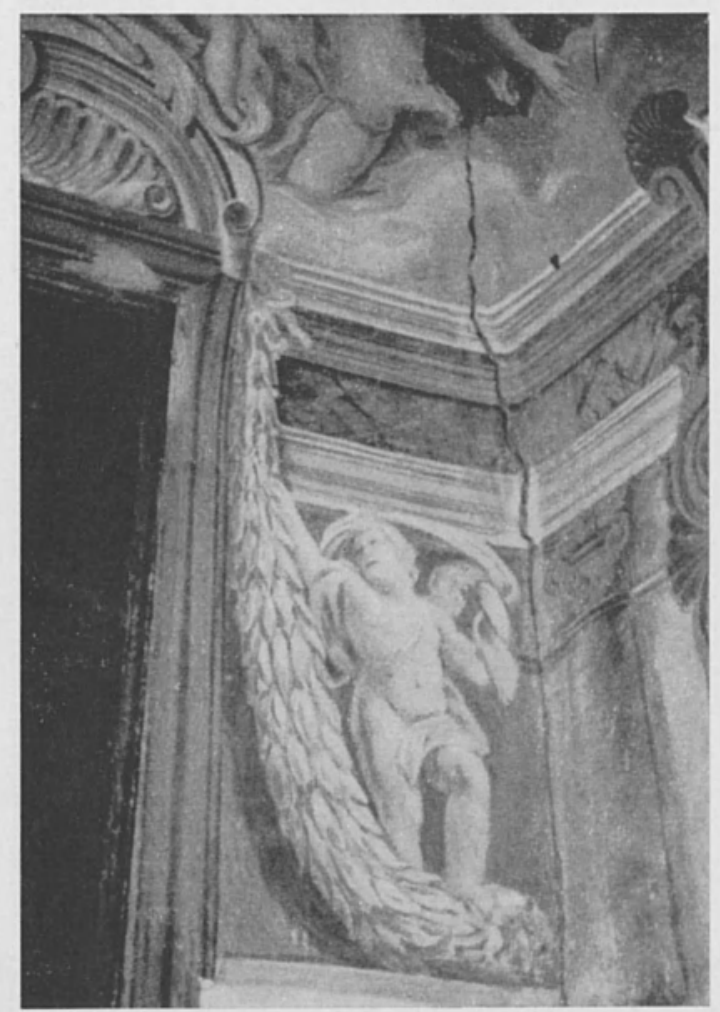

Fig. 5 - Castello di Rota. Lesioni nella volta e nelle murature laterali di una sala del primo piano.

(Foto G. Cirilli)

fenditure si sono aperte nelle murature divisorie ed anche in alcune di quelle perimetrali (Fig. 6); per ogni dove, cadute di calcinacci e di pezzi d'intonaco. Tutti gli altri edifici della borgata hanno riportato danni più o meno sensibili.

Il movimento sismico a Rota fu percepito nettamente come sussultorio, ebbe una durata molto breve (non più di $3-4$ secondi) e fu preceduto da un fortissimo rombo sotterraneo. 
L'altro centro abitato interno alla zona epicentrale, Civitella Cesi, è un paesino di circa 300 abitanti che, a quota $190 \mathrm{sul}$ mare, si eleva sopra un grosso sperone di tufo vulcanico situato in prossimità della

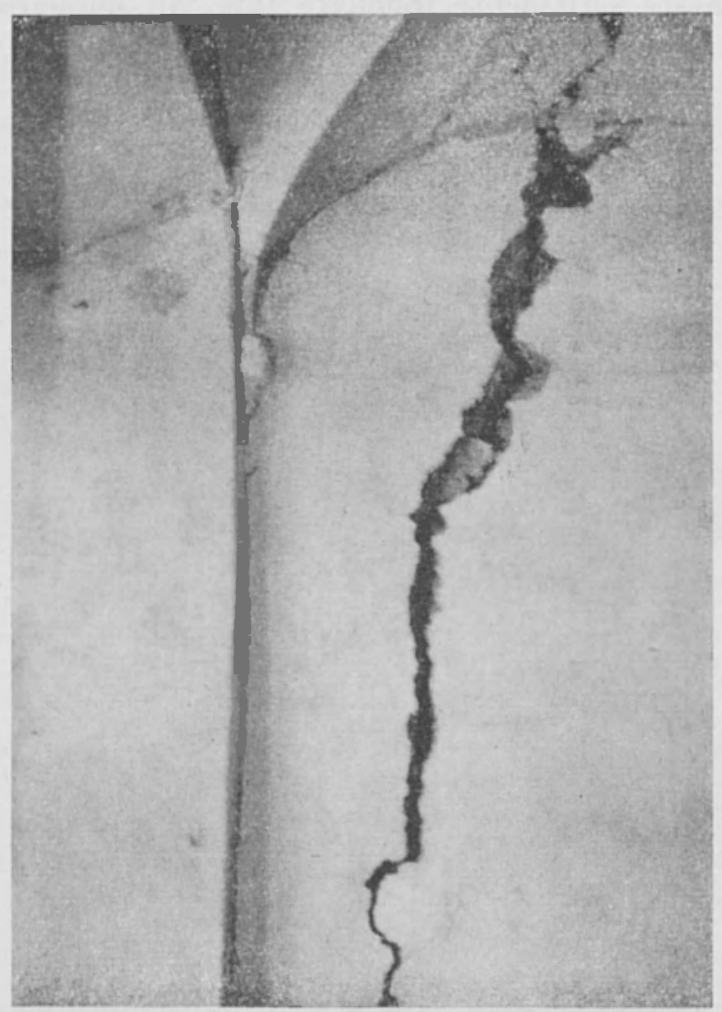

Fig. 6 - Castello di Rota. Grave lesione nella parete della scala che conduce dal $1^{\circ}$ al $2^{\circ}$ piano.

(Foto G. Cirilli)

confluenza del Fosso Borgonero col Torrente Vesca, tributario, quest'ultimo, del Fiume Mignone. All'estremità Sud-Est dell'abitato, costituito di case basse quasi tutte di antica e scadente costruzione, s'innalza un castello di origine medioevale, restaurato nella seconda metà del XVI secolo per opera del cardinale Federico Cesi (Fig. 7).

Anche a Civitella Cesi la scossa delle $9^{\mathrm{n}} 56^{\mathrm{m}}$ fu sentita con fortissima intensità (grado VII) e causò un enorme spavento nella popolazione che, presa dal panico per il ripetersi delle scosse, si riversò tutta nelle strade passando poi la notte all'aperto. I danni, tenuto conto della 
piccolezza del paese, debbono essere giudicati piuttosto rilevanti: 5 alloggi lesionati gravemente, 11 danneggiati mediamente e una quindicina lievemente; sensibili fenditure si sono prodotte nelle

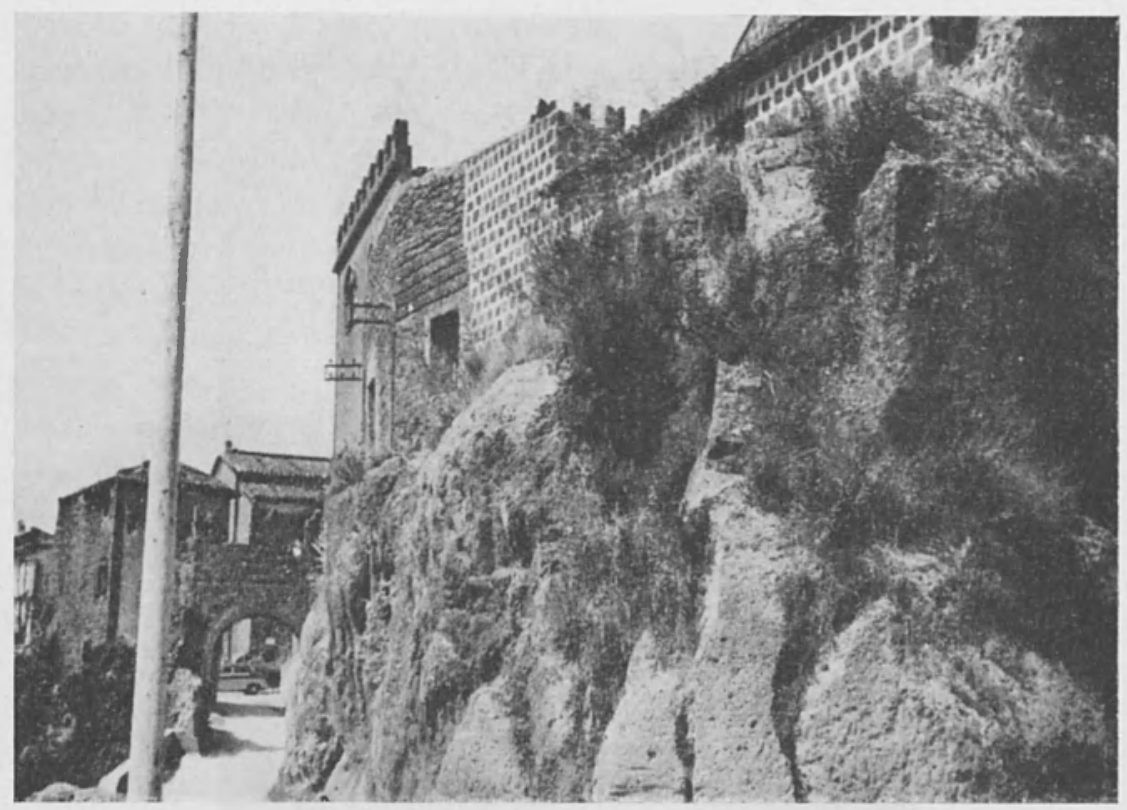

Fig. 7 - Il Castello di Civitella Cesi, poggiato, come tutte le case del paesino, su tufo vulcanico, ha subito sensibili lesioni in alcuni muri portanti e divisori.

(Foto G. Cirilli)

volte e nelle arcate della chiesa parrocchiale di $\mathrm{S}$. Leonardo; nel castello, oltre alla caduta di un comignolo e di una quantità di calcinacci, si sono avute lesioni di una certa entità in alcuni muri perimetrali e in alcune piattabande.

Le caratteristiche della scossa furono press'a poco uguali a quelle manifestatesi nel borgo di Rota: durata molto breve (non più di 4 secondi), movimento inizialmente sussultorio, preceduto da un pauroso boato e subito seguito da una sensibile ondulazione.

Le repliche di cui è stato dato poc'anzi l'elenco furono tutte avvertite sia a Rota che a Civitella Cesi.

Le notizie particolareggiate raccolte nella zona di più intenso scotimento e nelle località ad essa più vicine hanno reso possibile precisare la ubicazione dell'epicentro, che è da ritenere sia rimasta la 
stessa anche per le repliche che hanno seguito la scossa principale. Esso cade nella valle del Mignone entro il triangolo avente per vertici le alture di M. Chiusia, M. Monastero e M. Piantangeli; alle sue coordinate geografiche possono essere assegnati i valori

$$
\varphi=42^{\circ} 11^{\prime} \mathrm{N} \text { e } \lambda=12^{\circ} 00^{\prime} \mathrm{E} \text { (da Greenwich) }
$$

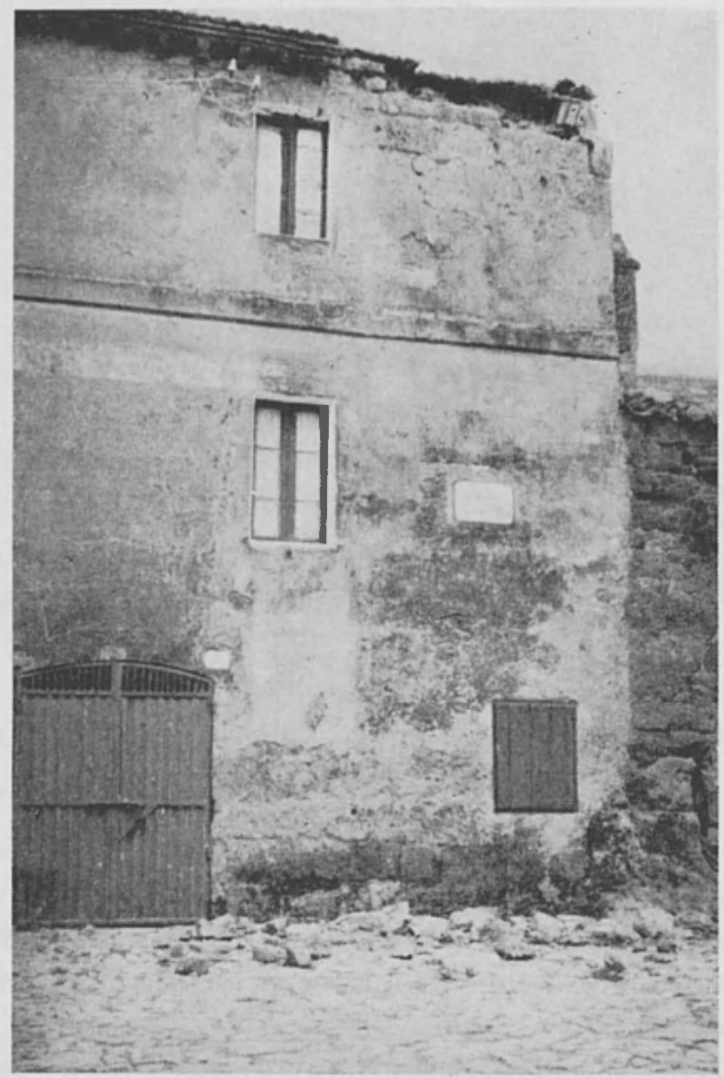

Fig. 8 - Civitella Cesi. Una casa in Piazza della Vittoria danneggiata dal terremoto.

(Foto G. Maiolo)

La zona epicentrale ha, a un dipresso, la forma di una ellisse poco eccentrica con l'asse maggiore, orientato approssimativamente da $\mathrm{NE}$ a $\mathrm{SW}$, di circa $10 \mathrm{~km}$ e l'asse minore di circa $8 \mathrm{~km}$; la sua area è quindi di circa $62 \mathrm{~km}^{2}$. 


\section{3. - AREa MaCROSISMiCA.}

L'area macrosismica relativa alla scossa principale, ossia l'area ove il moto tellurico è stato percepito sia pur lievemente dall'uomo, è di notevole ampiezza. Essa abbraccia infatti (Fig. 1) tutta la Tuscia romana, cioè la regione compresa fra il corso del Fiume Fiora, il Mar Tirreno e l'ultima parte del Tevere. La sua struttura orografica è caratterizzata principalmente dagli apparati vulcanici Sabatino, Cimino, Volsinio e, fra il Lago di Bracciano e il mare, dal gruppo delle colline in gran parte trachitiche della Tolfa.

Della zona di maggior scuotimento (grado VII) abbiamo già detto.

La isosista di grado VI, che racchiude la zona ove il sisma è stato sentito molto fortemente ed ha causato anche qualche danno è pur essa una curva press'a poco ellittica, eccentrica rispetto alla zona epicentrale, con l'asse maggiore, diretto ancora da NE a SW, della lunghezza di circa $30 \mathrm{~km}$ e l'asse minore di circa $16 \mathrm{~km}$. Entro questa zona, a Sud-Ovest dell'epicentro, s'imnalzano i Monti della Tolfa, culminanti a 628 metri nel Monte Urbano e costituiti principalmente da formazioni vulcaniche del Quaternario antico addossate a substrati sedimentari di età terziarie (calcari marnosi, argille gessifere mio-plioceniclre, arenarie calcaree, ecc.).

Nei due più popolosi centri dei Monti Tolfetani, Tolfa e Allumiere, costruiti ambedue su rocce trachitiche, l'uno a 470 e l'altro a 500 metri sul mare, la scossa delle $9^{\mathbf{n}} \mathbf{5 6}^{\mathrm{m}}$ fu sentita da tutti gli abitanti con grande spavento e anche in queste località essa fu preceduta da un rombo sotterraneo.

Lievi danni nella cittadina di Tolfa, assai prossima alla zona epicentrale: aggravamento di vecchie lesioni esistenti nel Palazzo comunale, fenditure leggiere in alcune case della parte vecchia dell'abitato, lesioni più o meno sensibili in fabbricati rurali delle campagne circostanti. Delle repliche, la prima venne sentita fortemente (V grado); quelle delle $10^{\mathrm{h}} 08^{\mathrm{m}}, 10^{\mathrm{h}} 21^{\mathrm{m}}$ e $10^{\mathrm{h}} 37^{\mathrm{m}}$ del 2 Luglio furono avvertite distintamente, ma con intensità più lieve che a Rota e a Civitella Cesi; appena percepite da poche persone, le successive. E stato riferito da abitanti del posto che, dopo le prime scosse, le acque di alcune sorgenti vicine presentarono temporanei intorbidamenti di colore rossastro.

Il paese di Allumiere, che, com'è noto, trae la sua origine ed il suo nome dalle cave di allumite scoperte nei dintorni nel 1462, sorge sul fianco meridionale del Monte delle Grazie, circa quattro chilometri 
ad Ovest di Tolfa. Gli effetti del terremoto sono stati qui piuttosto gravi nel Palazzo del Comune che, già da tempo in condizioni di stabilità molto precarie, ha subito notevoli lesioni nelle sue strutture murarie. Danni invece assai lievi si sono avuti in alcune abitazioni private. La replica delle $10^{\mathrm{h}} 03^{\mathrm{m}}$, quelle delle $10^{\mathrm{h}} 08^{\mathrm{m}}$ e $10^{\mathrm{h}} 21^{\mathrm{m}}$ furono distintamente sentite e contribuirono a creare uno stato di panico nella popolazione; le successive però risultarono leggerissime e qualcuna di esse non venne neanche avvertita.

Le osservazioni macrosismiche hanno messo in evidenza che il decremento d'intensità della scossa principale dal V $I 1$ al VI grado è stato meno rapido verso SW che nelle altre direzioni. Mentre, per esempio, a Blera, situata solo una decina di chilometri a Nord dell'epicentro, la scossa è risultata di V grado, nella città di Civitavecchia, distante circa il doppio, essa è giunta ancora molto forte così da suscitare, specie dopo le prime due repliche (le sole avvertite nella città), un allarme vivissimo in gran parte degli abitanti. La sua intensità peraltro certamente non ha superato il VI grado e giustifica questa valutazione il fatto che $i$ danni che si sono verificati in due fabbricati e ne hanno consigliato lo sgombero non sono stati altro che un aggravamento di lesioni da molti anni esistenti e già da tempo messe sotto controllo con biffe. Gli altri effetti del terremoto a Civitavecchia, per quanto abbiamo potuto vedere, si sono ridotti a cadute di calcinacci e a qualche fenditura in case di vecchia e difettosa costruzione.

Oltre che nelle località sopra menzionate, il sisma delle $9^{\mathrm{h}} 56^{\mathrm{m}}$ fu sentito di VI grado a Barbarano Romano, a Veiano e a Bagni di Stigliano. Anche in queste località la scossa venne preceduta da un boato e le repliche avvertite furono soltanto quelle delle $10^{\mathrm{h}} 03^{\mathrm{m}}$, $10^{\mathrm{h}} 08^{\mathrm{m}}$ e $10^{\mathrm{h}} 21^{\mathrm{m}}$.

A Barbarano Romano, paese dal caratteristico aspetto medioevale costruito sopra una rupe tufacea a picco su una profonda ed aspra gola incisa dal Torrente Biedano, si sono avute lievi lesioni in alcune case private, nella Chiesa parrocchiale di S. Maria Assunta, nella Chiesa di S. Michele e nella Canonica. Press'a poco dello stesso tipo e della stessa entità i danni verificatisi a Veiano in case vecchie, nel Duomo e in murature interne del cinquecentesco castello che sorge nella parte antica del paese.

La località Bagni di Stigliano, frazione del Comune di Canale Monterano è situata nella valle di un affluente del Mignone chiamato Fosso Lenta; vi ha sede soltanto uno stabilimento terapeutico per l'utilizzazione di acque sulfuree fredde e termali note sin dall'antichità. 
In questo luogo il terremoto ha lesionato un fabbricato poco distante dall'edificio alberghiero.

La zona dove la scossa delle $9^{\mathrm{h}} 56^{\mathrm{m}}$ venne sentita fortemente con le caratteristiche del $\mathrm{V}$ grado Mercalli si estende parte nella provincia di Roma e parte in quella di Viterbo. Essa è limitata da una isosista abbastanza regolare aperta sul Tirreno la quale, partendo dai pressi di S. Severa, passa a Sud di Castel Giuliano, poi ad Ovest del Lago di Bracciano, attraversa il Lago di Vico, indi piegando verso Occidente torna a mare in prossimità della foce del Mignone.

L'intera superficie racchiusa da detta isosista ha una estensione di circa $1100 \mathrm{~km}^{2}$, sicché il raggio $r_{5}$ del cerchio avente la stessa area è di $18,7 \mathrm{~km}$. Anche nella zona isosismica di $\mathrm{V}$ grado la scossa fu preceduta da un rombo, ove più ove meno intenso.

Dal V al III grado l'intensità è andata decrescendo in modo quasi uniforme nelle diverse direzioni sicché le isosiste di grado IV e III presentano un andamento presso che simile a quello della isosista di grado $\mathrm{V}$.

Non altrettanto regolare è la isosista di II grado, che presenta nella sua parte settentrionale delle sinuosità piuttosto accentuate. Questa isosista, che segna il limite della zona dove il movimento tellurico è stato percepito dalle persone, mostra chiaramente che l'estinzione macrosismica della scossa sulla terraferma è avvenuta molto più lentamente verso Nord che nelle altre direzioni. A Nord infatti la scossa ha mantenuto ancora la sua percettibilità a circa $60 \mathrm{~km}$ dall'epicentro, mentre nelle altre direzioni appare completamente estinta dopo distanze di 30-35 chilometri. Nell'area isosismica di II grado alcune località hanno avvertito il moto tellurico più intensamente del territorio circostante. Così, per esempio, a Montefiascone, la bella cittadina distesa sull'alto di un colle vulcanico a breve distanza dal Lago Volsinio, la scossa è stata distintamente sentita (IV grado), causando anche un certo allarme nella popolazione.

L'area totale della superficie scossa macrosismicamente è di circa $3550 \mathrm{~km}^{2}$.

Nella Tabella seguente riportiamo l'elenco dei luoghi ove è stata sentita la scossa principale, disposti in ordine d'intensità decrescente con l'indicazione della provincia e, se frazioni, anche del Comune di appartenenza. 


\begin{tabular}{|c|c|c|}
\hline $\mathbf{N}$ & Località & Intensità \\
\hline 1 & $\begin{array}{l}\text { Civitella Cesi (Viterbo - Fraz. del Comune di } \\
\text { Blera) }\end{array}$ & VII \\
\hline - 2 & Rota (Roma - Fraz. del Comune di Tolfa) & $"$ \\
\hline 3 & Allumiere (Roma) & VI \\
\hline 4 & $\begin{array}{l}\text { Bagni di Stigliano (Roma - Fraz. del Comune } \\
\text { di Canale Monterano) }\end{array}$ & $"$ \\
\hline 5 & Barbarano Romano (Viterbo) & VI \\
\hline 6 & Civitavecchia (Roma) & $"$ \\
\hline 7 & Tolfa (Roma) & $"$ \\
\hline 8 & Veiano (Viterbo) & ” \\
\hline 9 & $\begin{array}{l}\text { Aurelia (Roma - Fraz. del Comune di Civita- } \\
\text { vecchia) }\end{array}$ & V \\
\hline 10 & Bassano Romano (Viterbo) & $"$ \\
\hline 11 & Blera (Viterbo) & $»$ \\
\hline 12 & Canale Monterano (Roma) & " \\
\hline 13 & Capranica (Viterbo) & $"$ \\
\hline 14 & $\begin{array}{l}\text { Castel Giuliano (Roma - Fraz. del Comune di } \\
\text { Bracciano) }\end{array}$ & ” \\
\hline 15 & Cura (Viterbo - Fraz, del Comune di Vetralla) & " \\
\hline 16 & Manziana (Roma) & ” \\
\hline 17 & Monte Romano (Viterbo) & " \\
\hline 18 & $\begin{array}{l}\text { Montevirginio (Roma - Fraz. del Comune di } \\
\text { Canale Monterano) }\end{array}$ & ” \\
\hline 19 & Oriolo Romano (Viterbo) & $"$ \\
\hline 20 & Santa Marinella (Roma) & $"$ \\
\hline 21 & Sasso (Roma - Fraz. del Comune di Cerveteri) & $"$ \\
\hline 22 & Vetralla (Viterbo) & " \\
\hline 23 & Villa San Giovanni in Tuscia (Viterbo) & $"$ \\
\hline 24 & Ronciglione (Viterbo) & IV $-V$ \\
\hline 25 & Bracciano (Roma) & IV \\
\hline 26 & Caprarola (Viterbo) & $n$ \\
\hline
\end{tabular}




\begin{tabular}{|c|c|c|}
\hline $\mathrm{N}$. & Località & Intensità \\
\hline 27 & Cerveteri (Roma) & IV \\
\hline 28 & Mazzano Romano (Roma) & $"$ \\
\hline 29 & Montefiascone (Viterbo) & $"$ \\
\hline 30 & Piansano (Viterbo) & $"$ \\
\hline 31 & $\begin{array}{l}\text { San Martino al Cimino (Fraz. del Comune } \\
\text { di Viterbo) }\end{array}$ & " \\
\hline 32 & $\begin{array}{l}\text { Santa Severa (Roma - Fraz. del Comune di } \\
\text { Santa Marinella) }\end{array}$ & $"$ \\
\hline 33 & Sutri (Viterbo) & " \\
\hline 34 & VITERBO & $"$ \\
\hline 35 & Canepina (Viterbo) & II I \\
\hline 36 & Carbognano (Viterbo) & $"$ \\
\hline 37 & Ceri (Roma - Fraz. del Comune di Cerveteri) & $n$ \\
\hline 38 & Fabrica di Roma (Viterbo) & " \\
\hline 39 & Monterosi (Viterbo) & $"$ \\
\hline 40 & Tarquinia (Viterbo) & $"$ \\
\hline 41 & Trevignano Romano (Roma) & $"$ \\
\hline 42 & Tuscania (Viterbo) & $n$ \\
\hline 43 & Vallerano (Viterbo) & $"$ \\
\hline 44 & $\begin{array}{l}\text { Vigna di Valle (Roma - Fraz. del Comune di } \\
\text { Bracciano) }\end{array}$ & " \\
\hline 45 & Vignanello (Viterbo) & $"$ \\
\hline 46 & Anguillara Sabazia (Roma) & II \\
\hline 47 & Arlena di Castro (Viterbo) & ” \\
\hline 48 & Bagnoregio (Viterbo) & $"$ \\
\hline 49 & Calcata (Viterbo) & $"$ \\
\hline 50 & Canino (Viterbo) & $"$ \\
\hline 51 & Capodimonte (Viterbo) & $"$ \\
\hline 52 & Castel Sant'Elia (Viterbo) & $"$ \\
\hline 53 & Corchiano (Viterbo) & $"$ \\
\hline
\end{tabular}




\begin{tabular}{|c|c|c|}
\hline N. & Località & Intensità \\
\hline 54 & $\begin{array}{l}\text { Ladispoli (Roma - Fraz. del Comune di Cer- } \\
\text { veteri) }\end{array}$ & I I \\
\hline 55 & Làtera (Viterbo) & $"$ \\
\hline 56 & Marta (Viterbo) & ” \\
\hline 57 & Montalto di Castro (Viterbo) & $"$ \\
\hline 58 & Nepi (Viterbo) & $"$ \\
\hline 59 & Onano (Viterbo) & $"$ \\
\hline 60 & Palidoro (Fraz. del Conune di Roma) & $"$ \\
\hline 61 & Palo (Roma - Fraz. del Comune di Cerveteri) & $"$ \\
\hline 62 & Pitigliano (Grosseto) & $"$ \\
\hline 63 & San Lorenzo Nuovo (Viterbo) & $"$ \\
\hline 64 & Tessennano (Viterbo) & " \\
\hline 65 & Valentano (Viterbo) & $"$ \\
\hline 66 & Vitorchiano (Viterbo) & $"$ \\
\hline
\end{tabular}

\section{4. - Profondità ipocentralie.}

Abbiamo cercato di valutare la profondità dell'ipocentro della scossa principale mediante i dati a nostra disposizione, cioè i tempi di arrivo delle onde $P_{\sigma}$ ed $S_{\sigma}$ alle stazioni sismiche di Roma e di Monte Porzio Catone, che sono rispettivamente

$t_{1}=09^{\mathrm{h}} 55^{\mathrm{m} 53^{\mathrm{s}}}$ (tempo d'arrivo delle $P_{g}$ nell'Osserv. di Roma)

$t_{1}^{\prime}=09^{\mathrm{h}} 56^{\mathrm{m}} 00^{\mathrm{s}}, 5\left(\#, \quad S_{g}\right.$

$t_{2}=09^{\mathrm{n}} 55^{\mathrm{m}} 56^{\mathrm{s}}, 4\left(\right.$ " " $\quad P_{g}$ " Monte Porzio),

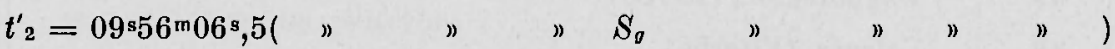

e assumendo come epicentro il punto di coordinate geografiche

$$
\varphi=42^{\circ} 11^{\prime} \mathrm{N} \text { e } \lambda=12^{\circ} 00^{\prime} \mathrm{E}
$$

che le accurate indagini macrosismiche ci fanno ritenere molto prossime al vero. 
Le distanze epicentrali $\Delta_{1}$ e $\Delta_{2}$ delle stazioni sismiche di Roma e di Monte Porzio sono state calcolate con una nota formula di trigonometria sferica e si sono ottenuti i valori

$$
\Delta_{1}=52 \mathrm{~km} \text { e } \Delta_{2}=70,5 \mathrm{~km} \text {. }
$$

Indicata con $h$ la profondità ipocentrale e con $v_{P g}$ la velocità delle onde $P_{q}$, semplici considerazioni sui triangoli formati dall'epicentro, dall'ipocentro e da ciascuna delle due stazioni di osservazione conducono alla relazione

$$
\sqrt{h^{2}+\Delta^{2}{ }_{2}}-\sqrt{h^{2}+\Delta^{2}}=v_{P g}\left(t_{2}-t_{1}\right)
$$

dalla quale, con facili passaggi si ricava la formula

$$
h=\sqrt{\left(\frac{\Delta_{2}{ }^{2}-\Delta^{2}{ }_{1}-k^{2}}{2 k}\right)^{2}-\Delta^{2}{ }_{1}}
$$

dove

$$
k=v_{P g}\left(t_{2}-t_{1}\right)
$$

Per la velocità di propagazione delle $P_{g}$ abbiamo assunto il valore $v_{P g}=5,39 \mathrm{~km} / \mathrm{sec}$ trovato da D. Di Filippo per la regione laziale. La profondità dell'ipocentro calcolata con la [1] risulta allora

$$
h=8,36 \mathrm{~km} \text {. }
$$

Molto concordanti con questo valore sono quelli che si ottengono con l'impiego delle velocità $v_{P g}$ e $v_{S_{g}}$ delle onde $P_{g}$ ed $S_{0}$ e dei tempi di arrivo $t_{P g}, t_{S g}$ di tali onde ad una medesima stazione di osservazione.

Calcolata la distanza ipocentrale $\Delta_{h}$ con la nota formula

$$
\Delta_{h}=K\left(t_{s g}-t_{P g}\right)
$$

dove

$$
K=\frac{v_{P g} \cdot v_{S g}}{v_{P g}-v_{S g}}
$$

e indicata con $\Delta$ la distanza epicentrale della predetta stazione, si può ottenere $h$ dalla formula

$$
h=\sqrt{\Delta^{2}{ }_{h}-\Delta^{2}}=\sqrt{\left[K\left(t_{S g}-t_{P g}\right)\right]^{2}-\Delta^{2}} .
$$


Assumendo, come si è detto, $v_{P_{g}}-5,39 \mathrm{~km} / \mathrm{sec}$ e $v_{s_{g}}=3,05 \mathrm{~km} / \mathrm{sec}$ (valore, anche quest'ultimo, trovato dal Di Filippo per la regione laziale) risulta

$$
K=7,0254 \text {. }
$$

Se allora applichiamo la [3] alla stazione sismica di Roma, per la quale

$$
t_{s g}-t_{P_{g}}=t_{1}^{\prime}-t_{1}=7^{\mathrm{s}, 5} \text { e } \quad \Delta=\Delta_{1}=52 \mathrm{~km}
$$

si ottiene

$$
h=8,50 \mathrm{~km} .
$$

Se l'applichiamo alla stazione sismica di Monte Porzio Catone, per la quale

$$
t_{s g}-t_{P g}=t_{2}^{\prime}-t_{2}=10^{\mathrm{s}, 1} \text { e } \Delta=\Delta_{2}=70,5 \mathrm{~km}
$$

abbiamo

$$
h=8,04 \mathrm{~km} .
$$

La media dei tre valori ottenuti è di $8,3 \mathrm{~km}$. Riteniamo pertanto che alla profondità dell'ipocentro possa essere attribuito il valore

$$
h=8 \mathrm{~km} \text { circa }
$$

che, a nostro avviso, è abbastanza in accordo con le osservazioni macrosismiche.

5. - ORA oRigine E ORA D'ARRIVo DeLLe $P_{g}$ ALL'EPICENtro.

Se indichiamo con $H$ l'ora origine, con $t_{P_{g}}$ l'ora d'arrivo delle $P_{g}$ ad una stazione di osservazione abbastanza vicina all'epicentro e con $\Delta_{h}$ la distanza ipocentrale di detta stazione, si ha evidentemente

$$
H=t_{P_{7}}-\frac{\Delta_{h}}{v_{P_{g}}} .
$$

Il calcolo di $\Delta_{h}$ mediante la [2] ci dà $\mathrm{km} 52,69$ per la stazione sismica di Roma e km 70,96 per quella di Monte Porzio. Introducendo questi valori nella [4] otteniamo in ambedue i casi identico risultato e precisamente

$$
H=09^{\mathrm{n}} 55^{\mathrm{m}} 43^{\mathrm{s}}, 2 .
$$


Determinate la profondità ipocentrale $h$ e l'ora origine $H$, è facile calcolare l'ora d'arrivo del movimento sismico all'epicentro. Essa risulta

$$
H_{E}=09^{\mathrm{n}} 55^{\mathrm{m}} 44^{\mathrm{s}}, \mathrm{i}
$$

\section{6. - Le manifestazioni sismiche nella "Tuscia Romana".}

Come si è visto nelle pagine precedenti, la propagazione macrosismica del terremoto studiato nella presente nota si estese tutta, fino ai limiti della sensibilità umana, entro la regione limitata dal Mar Tirreno, dal Fiume Fiora e dal corso inferiore del Tevere: è questa la regione, oggi appartenente in parte alla provincia di Roma e in parte a quella di Viterbo, che, per essere stata dapprima dominio degli Etruschi e poi dei Romani, venne nel Medioevo denominata "Tuscia Romana ". Di questa regione, nella quale, tra i Monti Sabatini e il litorale civitavecchiese, sorgono i Monti della Tolfa, esamineremo qui ora l'attività sismica per rilevare quali sono le zone di essa che appaiono più soggette ai movimenti tellurici.

Nella Tuscia Romana esistono due attive zone sismiche ben individuate da terremoti generalmente poco estesi, ma che talora hanno raggiunto una notevole intensità: esse sono la zona vulcanica circondante il Lago di Bolsena, di sismicità assai elevata, e quella, pure vulcanica ma di sismicità moderata, che si estende attorno al Lago di Vico. Ne passeremo in rassegna i principali centri di scotimento dando anche un cenno delle rispettive manifestazioni (Fig. 9).

Sul versante esterno settentrionale del grande apparato vulcanico dei Monti Volsini si trovano tre centri sismici: uno, in prossimità di Onano; un altro, nella zona compresa fra Grotte di Castro, San Lorenzo Nuovo e Acquapendente; il terzo, nei pressi di Castel Giorgio ( $\left.{ }^{1}\right)$. Tali centri sono molto vicini l'uno all'altro ed i frequenti terremoti da essi generati hanno tutti le caratteristiche di quelli d'origine vulcanica (piccola profondità ipocentrale e modesta, talvolta minima, estensione macrosismica).

( $\left.{ }^{1}\right)$ Tutte queste Iocalità sono classificate come sismiche di $2^{\text {a }}$ categoria nella legge 25 Novembre 1962, n. 1684 e in esse è obbligatoria l'osservanza delle norme tecniche di edilizia antisismica. 
All'attività del centro sismico di Onano si devono attribuire:

- due scosse di V grado avvenute durante l'anno 1923 (2 Luglio e 10 Dicembre);

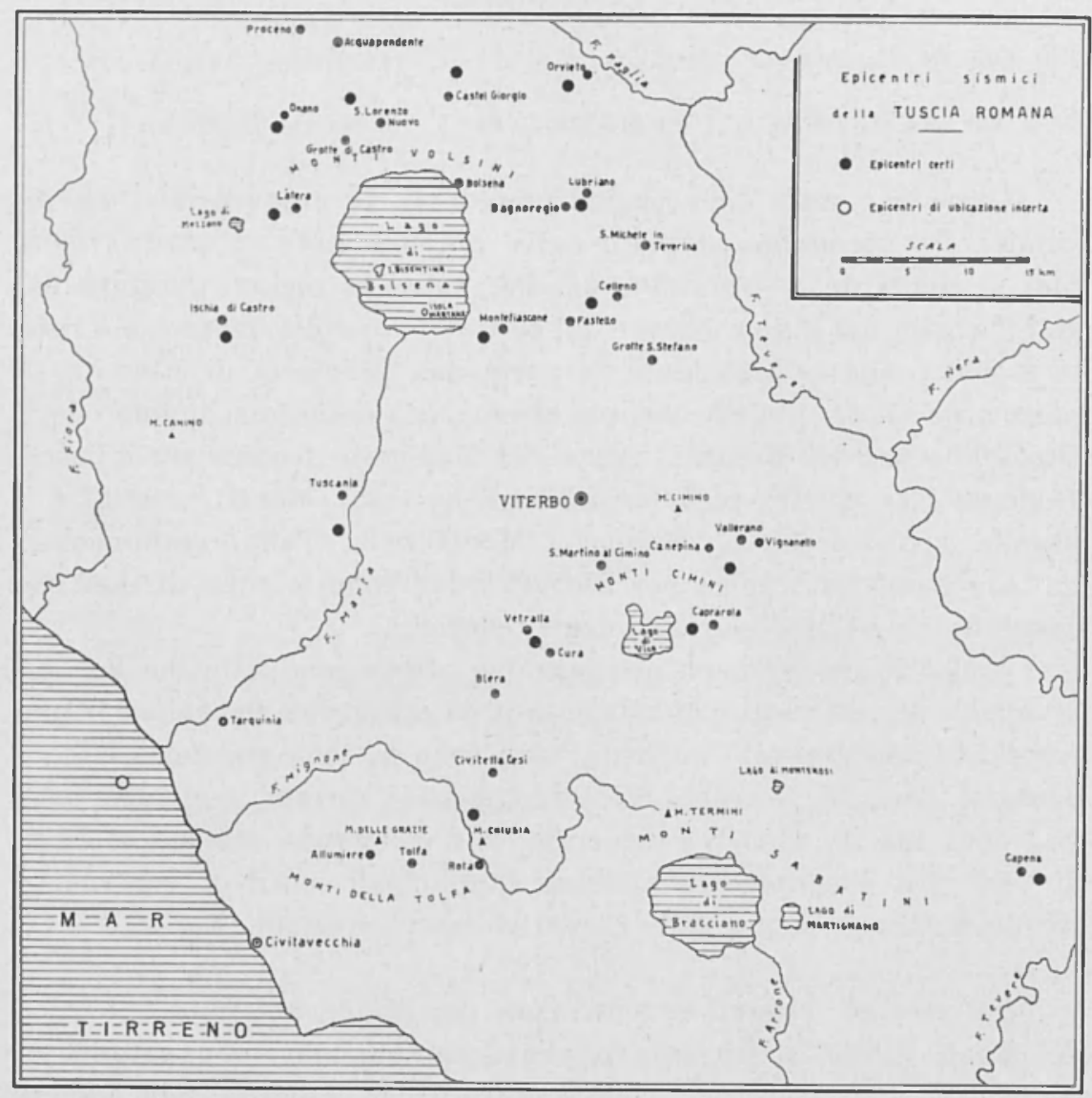

Fig. 9

- la scossa, pure di V grado, dell'8 Gennaio 1926 verificatasi qualche ora dopo un forte terremoto nel Monte Amiata;

- il brevissimo periodo sismico, con un massimo di VI grado, del 12-13 Dicembre 1929;

- quattro scosse di VI grado e due di V avvenute il 22 Dicembre 1930 a brevi intervalli di tempo l'una dall'altra, seguite da un'altra di V grado l'8 Gennaio 1931; 
- alcune scosse di mediocre intensità succedutesi nei giorni 12 e 13 Febbraio 1935;

- una scossa di V-VI grado del 15 Giugno 1936;

- una scossa di $\mathrm{V}$ grado avvenuta alle $00^{\mathrm{h}} 11^{\mathrm{m}}$ circa del 27 Agosto 1966 e seguita da alcune repliche di minore intensità fino alle $07^{\mathrm{h}}$ dello stesso giorno.

Dal secondo dei centri sismici sopra citati ebbero origine:

- un lungo periodo sismico costituito da numerose scosse non molto intense susseguitesi dall'11 Agosto al 18 Settembre 1888;

- una serie di scosse mediocri, con un massimo di $\mathrm{V}$ grado, sentite tutte specialmente ad Acquapendente e nel vicinissimo paese di Proceno nella mattina del 10 Maggio 1920;

- un violento periodo sismico svoltosi dal 29 Novembre al 4 Dicembre 1921, con due massimi di VII grado che causarono gravi danni nei paesi di Grotte di Castro e San Lorenzo Nuovo; una breve, ma molto forte ripresa dei fenomeni sismici il 27 Marzo 1922 con cinque scosse di VI grado avvenute tutte in detta giornata;

- un periodo sismico di breve durata (9 e 10 Aprile 1924), ma molto intenso, con due massimi di VII grado e tre scosse di VI che arrecarono molti danni negli abitati di Acquapendente, San Lorenzo Nuovo e Grotte di Castro;

- un altro periodo sismico, durato dal 18 al 21 Gennaio 1948, la cui scossa più forte (VI grado) fu sentita a San Lorenzo Nuovo più intensamente che altrove;

- due scosse fortissime (VI-VII grado) sentite ad Acquapendente e San Lorenzo Nuovo il 6 Dicembre 1957, dopo il terremoto quasi rovinoso di Castel Giorgio di cui ora brevemente diremo.

Dal focolare sismico prossimo a Castel Giorgio, mai rivelato precedentemente da manifestazioni di notevole intensità, irraggiò alle $05^{\mathrm{h}} 55^{\mathrm{m}}$ del 6 Dicembre 1957 un terremoto di VII-VIII grado che in detto paese produsse danni assai gravi. Il violento sisma fu seguito da numerose repliche di minore intensità fino all'11 Dicembre. È da credere che esso abbia provocato un risveglio del centro sismico di cui abbiamo dianzi parlato, poiché due intense scosse, avvenute rispetticamente alle $10^{\mathrm{h}} 18^{\mathrm{m}}$ e alle $23^{\mathrm{h}} \mathbf{5 6}^{\mathrm{m}}$ del 6 Dicembre, risultarono, come sopra si è detto, di VI-VII grado ad Acquapendente e a San Lorenzo Nuovo mentre vennero sentite a Castel Giorgio con intensità molto inferiore. 
Probabilmente dal centro di Castel Giorgio ebbe origine il breve e poco intenso periodo sismico iniziatosi alle $05^{\mathrm{h}} 04^{\mathrm{m}}$ del 18 Agosto 1962 e conclusosi alle $22^{\mathrm{h}} 30^{\mathrm{m}}$ circa del 20 Agosto. Si ebbero una decina di scosse quasi tutte di leggera e mediocre intensità. Solo due $\left(09^{\mathrm{h}} 58^{\mathrm{m}}\right.$ del giorno 18 e $01^{\mathrm{h}} 55^{\mathrm{m}}$ del giorno 19) furono sentite di $\mathrm{V}$ grado nella zona di Castel Giorgio e Castel Viscardo e più leggermente a Orvieto, Montefiascone, San Lorenzo Nuovo, Proceno, e Acquapendente.

Un terremoto avvenuto il 19 Gennaio 1901 indica inoltre l'esistenza di un centro sismico secondario anche a Nord-Est del Lago Volsinio, in vicinanza di Orvieto: esso infatti fu molto forte ed arrecò qualche danno solo in tale città, mentre venne avvertito debolmente nei paesi vicini.

Passando ora a considerare la zona ad oriente del Lago di Bolsena, dobbiamo anzitutto far menzione dell'importante centro sismico di Bagnoregio, la cui maggiore manifestazione è rappresentata dal disastroso terremoto del Giugno 1695. Precedute da diversi e forti movimenti sismici premonitori, la mattina dell'11 Giugno, con appena quindici minuti d'intervallo l'una dall'altra, sopravvennero le due terribili scosse principali. Rimasero devastate Bagnoregio e Lubriano e gravemente danneggiate numerose località ad Est del lago, specialmente Bolsena, Montefiascone, Grotte Santo Stefano, Celleno e San Michele in Teverina. A giudicare dalle notizie relative ai danni avvenuti nella zona epicentrale (territorio di Lubriano e Bagnoregio), il sisma dovette raggiungere un'intensità di VIII-IX grado almeno. Alla elevatissima intensità corrispose una vasta propagazione macrosismica: il movimento tellurico infatti venne avvertito anche a Roma e nei Castelli Romani, cioè ad una distanza di oltre 100 chilometri dall'epicentro.

Un terremoto di notevole intensità (VII grado) con epicentro nei pressi di Bagnoregio si è avuto anche al principio di questo secolo (21 Giugno 1903).

Due altri focolai sismici sono stati accertati nella zona volsinia ad Est del lago: uno è situato nelle immediate vicinanze di Montefiascone, l'altro nei pressi di Celleno. Del primo è da ricordare un terremoto di VII grado, molto localizzato, del 17 Ottobre 1917; del secondo, un terremoto di VI grado, anch'esso di carattere locale, del 13 Maggio 1931. Una recente manifestazione del centro sismico di Celleno si è avuta con una serie di sette scosse avvenute nei giorni 16 e 17 Agosto 1966: la prima alle $22^{\mathrm{h}} 10^{\mathrm{m}}$ del giorno 16 e l'ultima 
alle $09^{\mathrm{h}}$ circa del giorno successivo. Esse furono tutte d'intensità lieve, eccetto la seconda $\left(22^{\mathrm{h}} 30^{\mathrm{m}}\right.$ del giorno 16$)$ che sembra aver raggiunto il $\mathrm{V}$ grado, e vennero avvertite specialmente a Celleno e nel paesino di Fastello che dista da Celleno appena cinque chilometri.

Veniamo ora alla zona situata ad Ovest del Lago di Bolsena. Si ha qui un centro sismico nei pressi di Ischia di Castro, la cui esistenza, già indicata da alcune scosse del 21 Luglio 1889, venne confermata da un periodo sismico svoltosi dal 19 Settembre al 4 Ottobre 1909. Le manifestazioni di esso, tutte localizzate in detto paese e negli immediati dintorni, raggiunsero il loro massimo il 20 Settembre con una scossa di VI grado che arrecò lievi danni in alcune abitazioni.

Per completare l'enumerazione dei centri sismici esistenti nella zona del Lago di Bolsena, occorre far cenno di un complesso periodo sismico che dal Settembre 1881 durò fino al Febbraio 1882 e fu caratterizzato da diversi spostamenti dell'epicentro. Alcune scosse, all'inizio, ebbero origine dai pressi di Caprarola, nel versante Est dei Monti Cimini; poi altre, lievi, ne furono sentite a Viterbo; altre ancora, pure lievi, a Montefiascone. Una, molto forte, avvenuta il 20 Settembre, ebbe l'epicentro a Celleno. Le più importanti però, oltre che per l'intensità anche perchè rivelarono la probabile esistenza di due altri centri sismici, furono quella fortissima del 29 Novembre 1881, il cui epicentro sembra essere stato nei pressi di Tuscania, a Sud del Lago di Bolsena, e quella quasi rovinosa della fine di Gennaio 1882 con epicentro in prossimità di Làtera, ad Ovest del Lago medesimo.

La zona del Lago di Vico, come si è accennato, presenta una sismicità nettamente inferiore a quella della zona volsinia. I rari terremoti corocentrici in essa avvenuti sono stati originati da tre centri situati sui versanti esterni dell'apparato vulcanico vicano.

Uno di essi, di scarsa importanza, si trova ad Est, nei pressi di Caprarola, ed è noto soltanto per le scosse, già sopra citate, avvenute all'inizio del periodo sismico del 1881-1882.

Un altro è ad Ovest, presso Vetralla: alla sua attività, oltre a leggiere scosse di piccolissima propagazione come quella del 7 Dicembre 1959, sono da attribuire il terremoto del 23 Dicembre 1877 che raggiunse la sua maggiore intensità (VI grado) a Cura, frazione del Comune di Vetralla distante $3 \mathrm{~km}$ appena dal capoluogo, e il terremoto del 12 Gennaio 1949 che fu di VI grado a Vetralla, di V a S. Martino al Cimino e a Blera. 
Il terzo, infine, è situato a Nord-Est, in prossimità dei paesi di Canepina e Vallerano. Di questo centro sismico, già messo in luce da una scossa di V grado l'8 Settembre 1902, si è avuta una nuova recente manifestazione nel Settembre del 1963. Una dozzina di scosse si susseguirono a brevi intervalli di tempo nelle prime ore del giorno 2 e la più forte di esse, avvenuta alle $01^{\mathrm{h}} 24^{\mathrm{m}}$ circa, raggiunse il VII grado causando sensibili danni in molte vecchie case di Canepina, Vallerano e Vignanello. I fenomeni si ripetettero nel pomeriggio del giorno 5 ed una forte scossa, arvenuta alle $16^{\mathrm{h}} 32^{\mathrm{m}}$ circa e seguita da poche repliche leggiere, accentuò i danni prodotti nei paesi predetti dalle scosse del giorno 2 .

Nel quadro della sismicità della Tuscia romana va collocato anche il violento terremoto del 26 Maggio 1819, che fu sentito fortemente sul litorale tirrenico dalla foce del Fiume Fiora fino a Civitavecchia ed arrecò danni gravi nella città di Tarquinia: fra gli altri, il crollo di una cupola della navata centrale della bella chiesa romanica di S. Maria di Castello. Dubbia però è rimasta la ubicazione dell'epicentro di questo terremoto: probabilmente esso ebbe origine da un centro sismico sottomarino situato in prossimità del litorale tarquiniese.

L'attività sismica, che, come abbiam visto, risulta molto elevata nei monti Volsini ed alquanto inferiore nei Monti Cimini, diviene minima nei Monti Sabatini. Torna poi ad essere intensa nei Colli Albani, con i quali termina, a Sud-Est di Roma, l'allineamento dei quattro grandi apparati vulcanici laziali. La storia sismica non registra movimenti tellurici di una certa importanza che abbiano avuto i loro epicentri sui fianchi dell'ampio sistema vulcanico sabatino; solo ad oriente di esso, alla destra del Tevere, e più precisamente nei pressi di Capena, troviamo un focolare sismico secondario il quale, il 15 Febbraio 1915, dette origine ad un terremoto di VI grado, di carattere locale, che causò lievi danni nell'abitato di Capena.

Un'uguale tranquillità sismica, dai tempi cui risalgono notizie storicamente accertate, ha regnato nella zona che si estende dalle pendici occidentali dei Sabatini fin quasi al mare di Civitavecchia, cioè nella zona dei Monti della Tolfa. Il periodo sismico del Luglio 1969 pertanto, pur non essendo stato di grande intensità e di notevole durata, rappresenta un evento assai singolare per tale zona ed è interessante per aver rivelato un centro di scuotimento fino ad oggi non ancora conosciuto. 


\section{BIBLIOGRAFIA}

Baratta M., I terremoti d'Italia, Fr. Bocca, Torino (1901).

Cavasino A., I terremoti d'Italia nel trentacinquennio 1899-1933, Ist. Poligr. dello Stato (1935).

Calor P., Attività sismica in Italia nel decennio 1930-1939, Le Monnier, Firenze (1942).

Di Filippo D., Il terremoto di Cervara di Roma dell's Settembre 1941, Pubblic. dell'I.N.G., n. 97 (1943).

De Panfilis M., Attività sismica in Italia dal 1953 al 1957, "Ann. di Geofisica ", XII, I (1959). 